\title{
Radiation Therapy Outcome and Clinical Features of Duodenal-Type Follicular Lymphoma
}

\author{
Hansang Lee, $\mathrm{MD}^{1}$ \\ Dongryul Oh, MD, $\mathrm{PhD}{ }^{2}$ \\ Kyungmi Yang, MD2 \\ Young Hyeh Ko, MD, PhD ${ }^{3}$ \\ Yong Chan Ahn, MD, PhD2 \\ Won Seog Kim, MD, PhD ${ }^{1}$ \\ Seok Jin Kim, MD, PhD ${ }^{1,4}$
}

\begin{abstract}
${ }^{1}$ Division of Hematology-Oncology, Department of Medicine, ${ }^{2}$ Department of Radiation Oncology, ${ }^{3}$ Department of Pathology, Samsung Medical Center, Sungkyunkwan University School of Medicine, Seoul, ${ }^{4}$ Department of

Health Sciences and Technology, SAIHST,

Sungkyunkwan University, Seoul, Korea
\end{abstract}

\author{
Correspondence: Seok Jin Kim, MD, PhD \\ Division of Hematology-Oncology, \\ Department of Medicine, Samsung Medical \\ Center, Sungkyunkwan University School \\ of Medicine, 81 Irwon-ro, Gangnam-gu, \\ Seoul 06351, Korea \\ Tel: 82-2-3410-1766 \\ Fax: 82-2-3410-1754 \\ E-mail: kstwoh@skku.edu \\ Received April 2, 2018 \\ Accepted July 6, 2018 \\ Published Online July 10, 2018 \\ *Hansang Lee and Dongryul Oh contributed \\ equally to this work.
}

\begin{abstract}
Purpose
Duodenal-type follicular lymphoma (FL) is a rare variant of FL. There is still no consensus on the initial treatment, and clinical features including endoscopic findings are not familiar to most physicians. The objective of this study was to evaluate the outcome of patients who were initially treated with radiation therapy for duodenal-type FL.
\end{abstract}

\section{Materials and Methods}

We retrospectively analyzed 20 patients who were consecutively diagnosed with duodenaltype FL between 2008 and 2017. All patients received radiation therapy with curative intent.

\section{Results}

The median age of the patients was 52 years (range, 26 to 66 years), and females were predominant. Most patients $(n=18,90 \%)$ had stage I disease, and were diagnosed by a regular health examination in an asymptomatic state. The histological grade was one in 19 patients (95\%), and the endoscopic findings were diffuse nodular $(n=8)$, whitish granular $(n=8)$, and mixed pattern $(n=4)$. Radiation therapy was delivered to 17 patients with 24 Gy in 12 fractions, and to three patients with 30.6-36 Gy in 18 fractions. All patients were evaluated with endoscopy for response to radiation therapy, and complete response was achieved in 19 patients (95\%). At the time of analysis, all patients survived without any evidence of late toxicities related with radiation therapy.

\section{Conclusion}

Taken together, radiation therapy alone could be effective in controlling duodenal lesion. A further study with longer follow-up duration is warranted to confirm our findings.

Key words

Duodenum, Follicular lymphoma, Radiation therapy

\section{Introduction}

Duodenal-type follicular lymphoma (FL) is an extremely rare variant of FL among primary non-Hodgkin lymphomas of the gastrointestinal tract $[1,2]$. This disease entity was previously included in primary intestinal follicular lymphoma. However, it was prosed as a new subtype of FL in the World Health Organization (WHO) classification due to its distinctive and clinical features [3]. Although FL predominantly occurs as a nodal disorder and is a more common subtype of lymphoma in Western countries than in Asian countries, duodenal-type FL has been reported more frequently in Asian countries, especially in Japan [4,5]. Basically, it shares similar pathology findings with nodal FL including morphology and positivity for CD10 and Bcl-6 immunohistochemistry [6]. However, its clinical features are closer to mucosa-associated lymphoid tissue (MALT) lymphoma rather than nodal FL because it is usually confined to the duodenum as a localized disease [5]. Indeed, a comprehen- 
sive gene expression analysis showed that gene expression profiles of duodenal-type FL were more closely related with MALT lymphoma than nodal FL [7]. Accordingly, radiation therapy (RT) is the first consideration for newly diagnosed duodenal-type FL in many institutes similar to limited stage disease of nodal FL where irradiation as preferred treatment option for localized disease [8,9]. A recent report of single center experience with 21 cases of duodenal-type FL also demonstrated that RT could be an effective initial treatment for this disease entity [10]. Nevertheless, there is still no consensus on the initial treatment for duodenal-type FL or the optimal timing and target volume of RT. Because of its low incidence, few data are available on the treatment outcome of patients who were treated with RT. Furthermore, the clinical features and endoscopic findings of patients with duodenal-type FL are still not familiar to most physicians. Therefore, in this study, we analyzed patients with duodenal-type FL who were consecutively treated with RT and report their clinical features including endoscopic findings and the treatment outcome.

\section{Materials and Methods}

\section{Patients}

A total of 20 patients were diagnosed with duodenal-type FL at the Samsung Medical Center between 2008 and 2017. Endoscopic biopsy was performed in all patients and they were diagnosed by an expert pathologist for lymphoma (Y.H.K). Staging work-up was done including physical examination, laboratory studies, computed tomography (CT) scans of thorax and abdomen, 2-deoxy-2-fluorine-18-fluoroD-glucose positron emission tomography integrated with CT $\left({ }^{18} \mathrm{~F}-\mathrm{FDG}\right.$ PET/CT) scan, and bilateral bone marrow biopsy and aspiration. Colonoscopy was done at the time of diagnosis; however, double-balloon enteroscopy or capsule endoscopy was not performed. After staging work-up, patients received involved-site RT with curative intent. Each patient fasted for at least 8 hours before the simulation. The planning CT scan was acquired in a supine position during free breathing. For all patients, a four-dimensional CT (4D $\mathrm{CT}$ ) was acquired at our institution. In $4 \mathrm{D} \mathrm{CT}$, the movement of the third part of the duodenum craniocaudally ranged from 0.75 to $2 \mathrm{~cm}$. The clinical target volume (CTV) included the whole duodenum. The internal target volume (ITV) for the CTV was delineated. The planning target volume (PTV) was defined with a margin of $1-1.5 \mathrm{~cm}$ to the CTV or ITV. A three-dimensional conformal RT technique was used in 13 patients and intensity-modulated RT (IMRT) in seven patients. One-arc volumetric modulated arc therapy technique was used for IMRT planning. Basically, the goal of planning the radiation field was that $95 \%$ of PTV should be covered by $99 \%$ of prescribed dose. Furthermore, a volume greater than one cubic centimeter does not receive more than $107 \%$ of the prescribed dose. Before 2013, RT was delivered with 30-36 Gy. Thereafter, RT was delivered with 24 Gy in 12 fractions. Chemotherapy was administered as an initial regimen for one patient. After the completion of RT, followup endoscopic examination was carried out to confirm complete response (CR). During follow-up, endoscopy and/or surveillance $\mathrm{CT}$ scan were regularly performed to monitor the occurrence of local and systemic relapse. We performed a follow-up evaluation every 3 to 4 months during the first year. After that, the follow-up interval was 6 months to 1 year.

\section{Study methods}

We retrospectively reviewed the medical records of all patients with duodenal-type FL to analyze their clinical features and treatment outcome. The pathology report, including the WHO grade of FL, was reviewed to confirm the diagnosis of FL. The staging was based on the Lugano classification for gastrointestinal tract lymphoma, and the prognostic group was determined by the International Prognostic Index (IPI) and Follicular Lymphoma International Prognostic Index (FLIPI) [11]. We also reviewed endoscopic findings of all patients to categorize the duodenal involvement pattern of FL. Information concerning RT-related toxicities, relapses, and outcomes was collected in a review of medical records. The last survival status was updated at the time of analysis in March 2018, and overall and progression-free survival outcomes were analyzed.

\section{Ethical statement}

All aspects of the study were approved by the Institutional Review Board of Samsung Medical Center (IRB No. 201708-002), and informed consent was exempted due to the retrospective nature of this study.

\section{Results}

\section{Characteristics of patients at diagnosis}

The median age of patients at diagnosis was 52 years (range, 26 to 66 years); 16 patients were $\leq 60$ years (Table 1 ). Females were predominant $(\mathrm{n}=14,70 \%)$, and the majority of 
Table 1. Patient characteristics at diagnosis

\begin{tabular}{|c|c|}
\hline Characteristic & No. $(\%)$ \\
\hline \multicolumn{2}{|l|}{ Age (yr) } \\
\hline$\leq 60$ & $16(80)$ \\
\hline$>60$ & $4(20)$ \\
\hline \multicolumn{2}{|l|}{ Sex } \\
\hline Male & $6(30)$ \\
\hline Female & $14(70)$ \\
\hline \multicolumn{2}{|c|}{ Performance status } \\
\hline ECOG 0/1 & $20(100)$ \\
\hline $\mathrm{ECOG} \geq 2$ & 0 \\
\hline \multicolumn{2}{|c|}{ Abdominal pain } \\
\hline Absence & $17(85)$ \\
\hline Presence & $3(15)$ \\
\hline \multicolumn{2}{|l|}{ Lugano stage } \\
\hline I & $18(90)$ \\
\hline II1 & $1(5)$ \\
\hline II2 & 0 \\
\hline IV & $1(5)$ \\
\hline \multicolumn{2}{|c|}{ Hemoglobin (g/dL) } \\
\hline$\geq 12$ & $18(90)$ \\
\hline$<12$ & $2(10)$ \\
\hline \multicolumn{2}{|l|}{ Serum LDH } \\
\hline Normal & $19(95)$ \\
\hline Elevated & $1(5)$ \\
\hline \multicolumn{2}{|c|}{ Bone marrow involvement } \\
\hline Absence & $19(95)$ \\
\hline Presence & $1(5)$ \\
\hline \multicolumn{2}{|c|}{ WHO histological grade } \\
\hline 1 & $19(95)$ \\
\hline 2 & 0 \\
\hline $3 \mathrm{~A}$ & $1(5)$ \\
\hline $3 B$ & 0 \\
\hline \multicolumn{2}{|l|}{ IPI risk (score) } \\
\hline Low $(0 / 1)$ & $15 / 5(100)$ \\
\hline \multicolumn{2}{|c|}{ FLIPI risk (score) } \\
\hline Low $(0 / 1)$ & $13 / 7(100)$ \\
\hline
\end{tabular}

ECOG, Eastern Cooperative Oncology Group; LDH, Lactate dehydrogenase; WHO, World Health Organization; IPI, International Prognostic Index; FLIPI, Follicular Lymphoma International Prognostic Index.

patients ( $\mathrm{n}=18,90 \%)$ had stage I disease confined to the duodenum. Only one patient had evidence of bone marrow involvement; however, only 5\% of cells were positively stained for CD20 and morphologically consistent with lymphoma in the tissue sample of bone marrow biopsy. This case with bone marrow involvement had grade 1 disease, and the flow cytometry of bone marrow aspirates did not show any evidence of lymphoma. As we could not determine its clini- cal significance, we treated the patient like other patients with stage I. Most patients ( $\mathrm{n}=15,75 \%)$ were asymptomatic at diagnosis and were incidentally diagnosed as part of a regular preventive medical examination. Only three patients complained of mild abdominal discomfort and dyspepsia at diagnosis. The elevation of serum lactate dehydrogenase (LDH) was observed in only one case; thus, all patients were scored as low risk on the IPI. FLIPI scoring also showed that all patients were low risk ( 0 to 1 ) (Table 1 ). Nineteen patients were grade 1 in the WHO histological classification (95\%), and the remaining patient was diagnosed as grade $3 \mathrm{~A}$. The staging work-up with CT scan and PET/CT showed no evidence of wall thickening or distant metastasis involving other organs such as gastrointestinal tract, liver, and spleen. The ${ }^{18} \mathrm{~F}$-FDG avid lesion of the primary site was found only in two patients (patient 8 and 12) with $>5.0$ of standardized uptake value (Deauville score 4) (Fig. 1). Thus, most patients had localized disease although the whole intestine was not evaluated by capsule endoscopy or double-balloon enteroscopy.

\section{Endoscopic finding of the primary lesion}

Primary lesions were confined to the duodenum in all cases; however, their gross appearance was variable from multiple polypoid to multiple granular lesions. The size of polypoid lesions was also variable from small nodules to 2 $\mathrm{cm}$-sized polyps, and the color and texture of all lesions were mainly a whitish granular pattern (Fig. 2). Thus, according to the endoscopic findings, the pattern of involvement was classified as follows: diffuse nodular $(\mathrm{n}=8)$, whitish granular $(n=8)$, and mixed pattern $(n=4)$ (Fig. 2). The site most commonly involved was the second portion of the duodenum $(\mathrm{n}=19)$; the second and third portions of the duodenum were involved in one patient.

\section{Treatment and survival outcome}

Among 20 patients, 19 patients received initially RT after diagnosis. One patient who was diagnosed in 2008 received 6 cycles of CVP (cyclophosphamide, vincristine, and prednisone) chemotherapy after diagnosis. However, the followup endoscopy and biopsy demonstrated disease progression 1.6 years after the completion of chemotherapy. Thus, he received RT as a salvage treatment. As a result, seventeen patients received RT with 24 Gy in 12 fractions, and three patients received 30-36 Gy with conventional fractionations. RT response was evaluated with endoscopy, and CR was achieved in 19 cases. We performed endoscopic biopsy to confirm pathological $\mathrm{CR}$, and pathological examination of biopsy samples showed no evidence of disease. However, one patient (patient No. 6) (Fig. 3A) showed partial response 
A

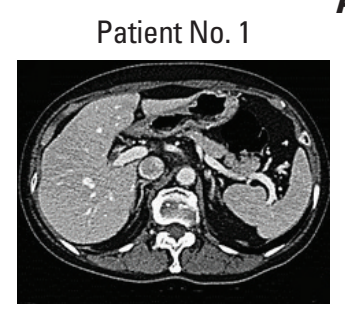

E

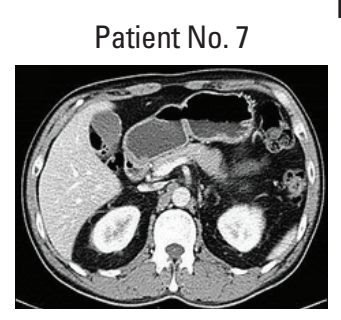

I

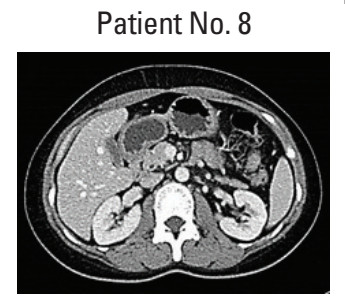

B

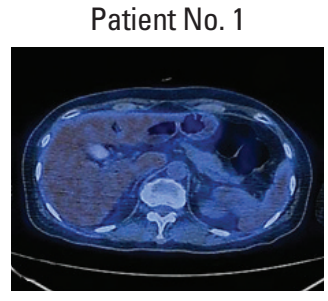

C

Patient No. 9

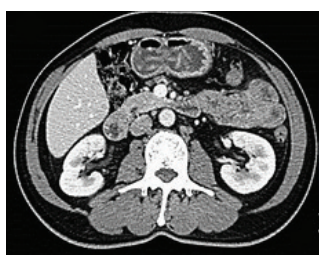

G

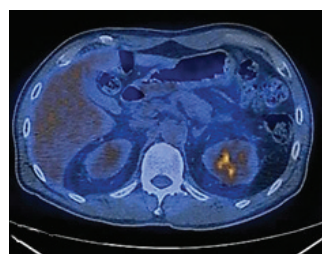

J

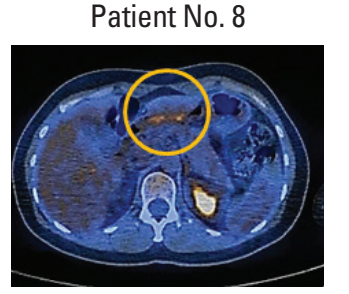
Patient No. 11

$\mathbf{F}$

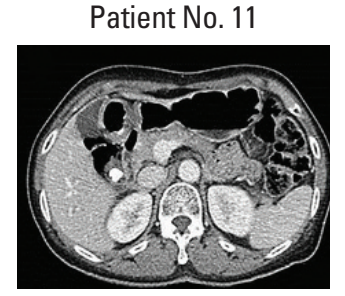

D

Patient No. 9

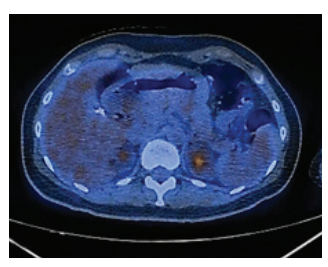

Fig. 1. Initial computed tomography $(\mathrm{CT})$ and positron emission tomography (PET)/CT scan imaging. (A, C, E, G, I, K) Most patients do not have severe wall thickening in the CT scan. (B, D, F, H) 2-Deoxy-2-fluorine-18-fluoro-D-glucose (FDG) uptake is not increased at the primary tumor site in the PET/CT. (J, L) Only two patients show increased FDG uptake (yellow circle).

on endoscopy at the 3-month follow-up after RT (Fig. 3B). Biopsy specimen also revealed residual tumor. This lesion was still persistent on re-biopsy at 6 and 9 months after RT (Fig. 3C and D). Finally, irradiation of 20 Gy was delivered to this patient, again. After that, the patient achieved CR and maintained the response. Another patient (patient No. 14) (Fig. 3E) achieved CR in duodenal lesion showed CR (Fig. 3E), but the colonoscopy revealed nodular lesions in the terminal ileum (Fig. 3G and H). The biopsy showed the presence of FL in the terminal ileum, thus, six cycles of rituximabcyclophospha-mide, vincristine, and prednisone (R-CVP) were performed. CR was achieved after chemotherapy, and the patient was alive without evidence of disease (Fig. 3I). The patient (patient No. 9) (Figs. 1C, D and 2C, D) with stage IV disease (bone marrow involvement) also achieved CR after RT and maintained CR at the time of analysis without evidence of systemic progression. We have only one patient who had initially serum LDH elevation (602 IU/L; normal range, 240 to $480 \mathrm{IU} / \mathrm{L}$ ) (Table 1). Three months after the completion of RT in this patient, the serum level of $\mathrm{LDH}$ was recovered to $305 \mathrm{IU} / \mathrm{L}$. At the time of analysis, only two patients who were initially treated with RT experienced disease progression or relapse after the completion of RT (S1 Fig.). All acute toxicities related with RT were grade 1 and most of these patients experienced mild nausea $(n=15)$. Other symptoms were abdominal pain or discomfort $(\mathrm{n}=7)$, dyspepsia $(n=3)$, fatigue $(n=3)$, anorexia $(n=2)$, vomiting $(n=2)$, and diarrhea $(n=2)$. No grade 2 or greater acute toxicities were observed during RT and over the follow-up period. The toxicities of patients receiving second RT and R-CVP chemotherapy were not different from that of remaining patients receiving $\mathrm{RT}$ alone. The median follow-up time was 20 months (range, 11 to 110 months) because 13 patients (65\%) were diagnosed between 2015 and 2017. At the time of analysis, all patients survived without any evidence of disease relapse and late toxicities related with RT. 
A

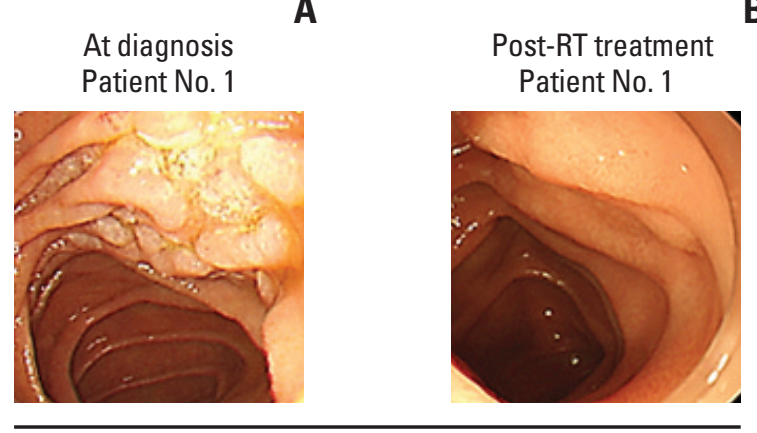

Diffuse nodular pattern

E

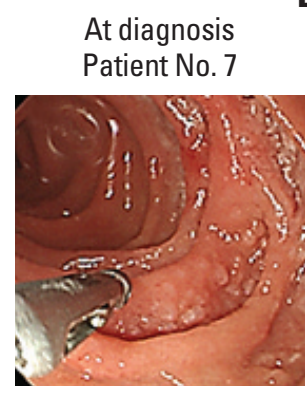

Post-RT treatment Patient No. 7

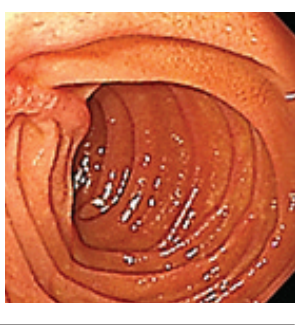

Diffuse nodular pattern

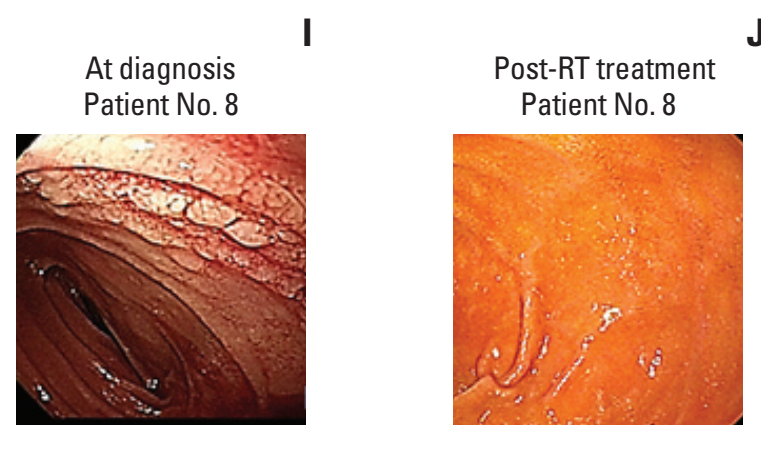

Diffuse nodular pattern
B

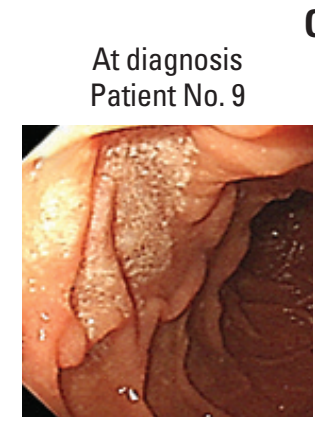

C

D

Post-RT treatment

Patient No. 9

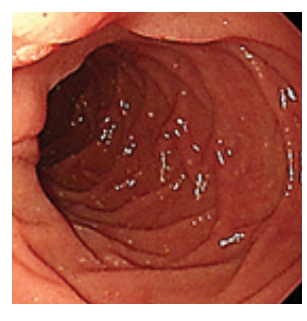

Whitish granular pattern

G

H

Post-RT treatment

Patient No. 11
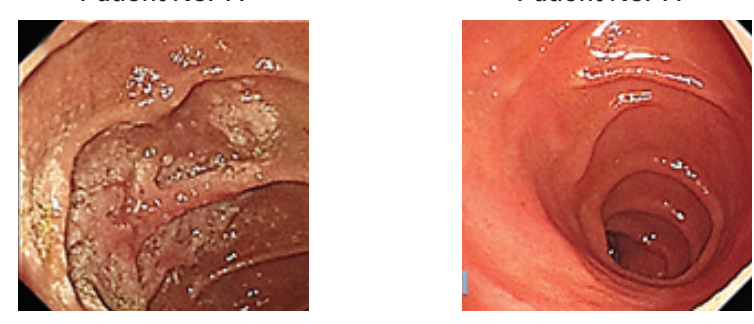

Whitish granular pattern

K

At diagnosis

Patient No. 12

Post-RT treatment

Patient No. 12
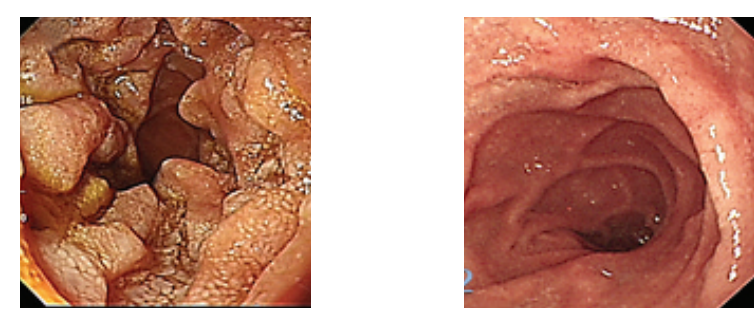

Whitish granular pattern

Fig. 2. Endoscopic findings of duodenal-type follicular lymphoma consist of diffuse nodular (A, E, I) and a whitish granular pattern $(C, G, K)$. These lesions disappear after radiation therapy (RT) (B, D, F, H, J, L).

\section{Discussion}

Duodenal-type FL is known as having a different pathogenesis to that of nodal FL. Thus, the biology of duodenaltype FL may be different although the initial event with the occurrence of $t(14 ; 18)(q 32 ; 21)$ in B cells of the duodenal mucosa was the same as that of nodal FL. Additional major oncogenic events might be absent in duodenal-type FL, and this might be related with the rarity of systemic dissemination and large cell transformation whereas most nodal FL can acquire additional genetic changes resulting in widespread disease [12,13]. The expression of a mucosal adhesion molecule, $\alpha 4 \beta 7$, was also demonstrated in duodenal-type FL, but not in nodal FL, which may increase the likelihood of localized disease in duodenal-type FL [14]. Similarly, duodenal- 
A

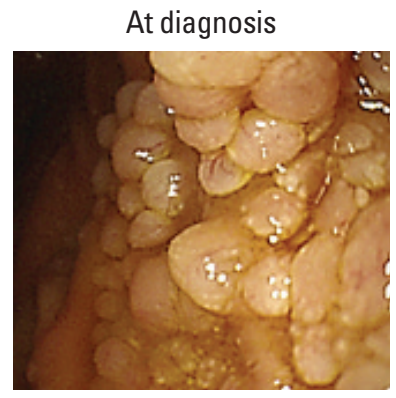

D

9 Months after RT

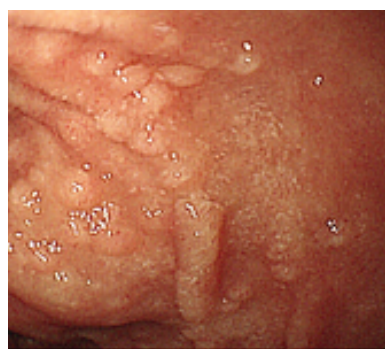

G

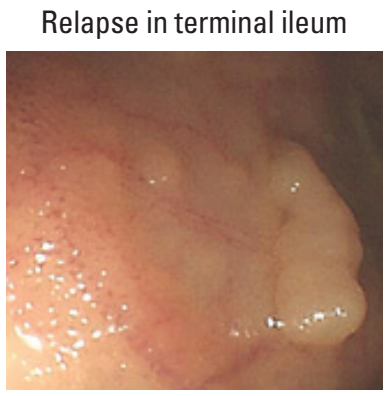

B

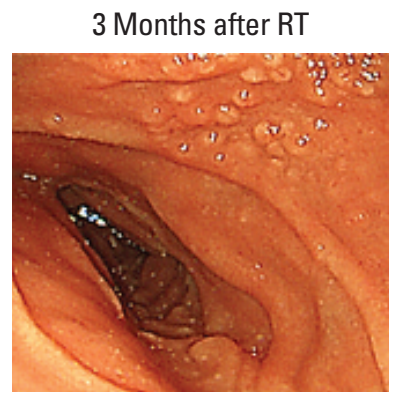

E

At diagnosis

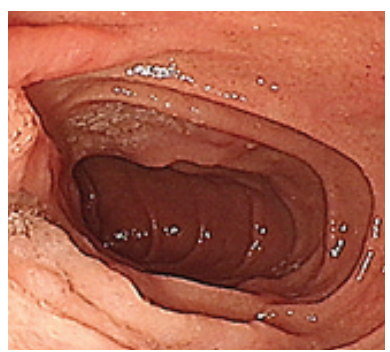

H

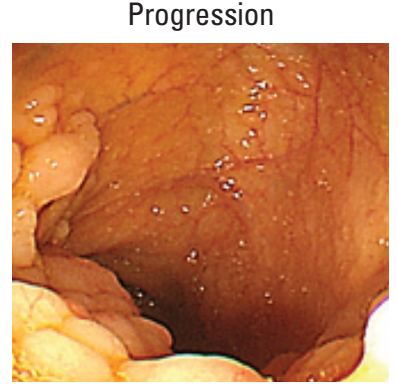

C

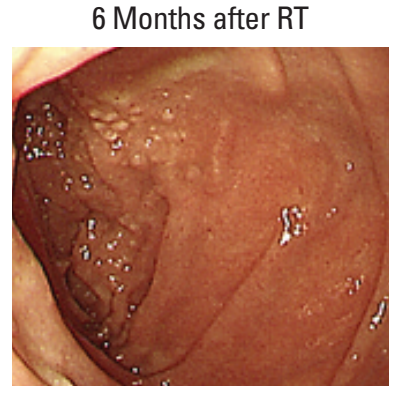

$\mathbf{F}$

3 Months after RT

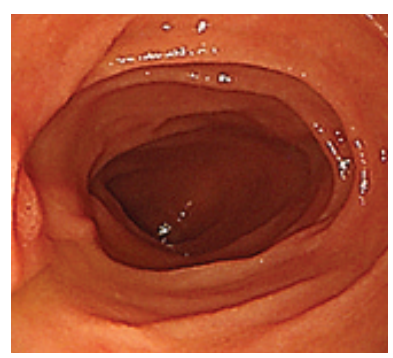

I

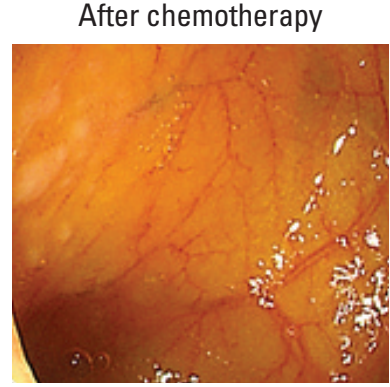

Fig. 3. (A) Initial lesions of patient No. 6. (B) Improved lesions on endoscopy at 3-month follow-up after radiation therapy. Biopsy also reveals residual tumor. (C) Endoscopic examination at 6-month follow-up shows no significant change. (D) At 9-month follow-up, the lesion progresses, and re-biopsy shows persistent disease. Finally, re-irradiation of 20 Gy is carried out on this patient. (E) Initial lesions of patient No. 14. (F) Complete response on endoscopy at 3-month follow-up after radiation therapy. (G) Relapse outside the radiation field. Colonoscopy reveals nodular lesions in the terminal ileum. Biopsy proves the presence of follicular lymphoma. (H) Follow-up colonoscopy shows progressive disease. (I) After six cycles of rituximab-cyclophosphamide, vincristine, and prednisone, complete response is achieved, and the patient is alive without evidence of disease.

type FL and MALT lymphoma have strong expression of macrophage inflammatory protein-3 $\alpha$ (CCL20) and its receptor, CCR6 [7]. The chronic inflammatory reaction induced by CCL20 in the gastrointestinal tract might influence the occurrence of duodenal-type FL and MALT lymphoma.

The comparison of our study with previous Japanese and
European retrospective cohorts showed several points of difference $[10,13]$. First, the median age of our study was younger. The reason for our younger sample might be influenced by the early detection of duodenal lesions by endoscopy in Korea because most Koreans can receive endoscopic examinations under the government-sponsored annual health 
Table 2. Comparison of our study results with previous studies

\begin{tabular}{|c|c|c|c|}
\hline Characteristic & $\begin{array}{l}\text { Our study } \\
\qquad(n=20)\end{array}$ & $\begin{array}{l}\text { Harada et al. [10] } \\
\qquad(n=21)\end{array}$ & $\begin{array}{l}\text { Schmatz et al. [13] } \\
\qquad(n=63)\end{array}$ \\
\hline Country & Korea & Japan & Germany/Austria \\
\hline Period & $2008-2017$ & $2005-2013$ & $1997-2008$ \\
\hline Follow-up (mo) & 20.0 & 43.2 & 77.0 \\
\hline Age (yr) & $52(26-66)$ & $62(46-79)$ & $65(32-83)$ \\
\hline \multicolumn{4}{|l|}{ Sex } \\
\hline Male & $6(30)$ & $6(29)$ & $31(49)$ \\
\hline Female & $14(70)$ & $15(71)$ & $32(51)$ \\
\hline \multicolumn{4}{|l|}{ Abdominal pain } \\
\hline Absent & $17(85)$ & $19(90)$ & $22(35)$ \\
\hline Present & $3(15)$ & $2(10)$ & $41(65)$ \\
\hline \multicolumn{4}{|l|}{ Lugano stage } \\
\hline I & $18(90)$ & $20(95)$ & $63(100)$ \\
\hline II1/II2/IV & $1(5) / 0 / 1(5)$ & $1(5) / 0 / 0$ & \\
\hline \multicolumn{4}{|l|}{ Histological grade } \\
\hline 1 & $19(95)$ & $17(81)$ & $63(100)$ \\
\hline 2 & 0 & $4(19)$ & \\
\hline $3 \mathrm{~A} / 3 \mathrm{~B}$ & $1(5) / 0$ & & \\
\hline \multicolumn{4}{|l|}{ IPI risk } \\
\hline Low & $20(100)$ & $21(100)$ & Not reported \\
\hline \multicolumn{4}{|l|}{ FLIPI risk } \\
\hline Low & $20(100)$ & $19(90)$ & $62(98)$ \\
\hline Intermediate & 0 & $2(10)$ & $1(2)$ \\
\hline \multicolumn{4}{|l|}{ Duodenal location } \\
\hline 2nd portion & $19(95)$ & 15 & Not specified \\
\hline 3rd portion & 0 & 3 & \\
\hline 2nd and 3rd portions & $1(5)$ & 2 & \\
\hline 2nd and 4 th portions & 0 & 1 & \\
\hline \multicolumn{4}{|l|}{$\mathrm{PET} / \mathrm{CT}$} \\
\hline Positive & 2 & 7 & Not reported \\
\hline Negative & 17 & 12 & \\
\hline \multicolumn{4}{|l|}{ Initial treatment } \\
\hline Radiation therapy & $19(95)$ & $21(100)$ & $19(30)$ \\
\hline Watch and wait & & & $24(38)$ \\
\hline Rituximab & & & $5(8)$ \\
\hline Chemotherapy ${ }^{\mathrm{a})}$ & $1(5)$ & & $8(13)$ \\
\hline \multicolumn{4}{|l|}{ Radiation therapy } \\
\hline Dose (Gy) & $24-36$ & $30-36$ & $30-45$ \\
\hline $\mathrm{CR} / \mathrm{PR}$ & $19 / 1$ & Not reported & $19 / 0$ \\
\hline Local progression & 1 & 0 & \\
\hline Systemic progression & 1 & 4 & \\
\hline Death & 0 & 0 & 0 \\
\hline
\end{tabular}

Values are presented as median (range) or number (\%). IPI, International Prognostic Index; FLIPI, Follicular Lymphoma International Prognostic Index; PET/CT, positron emission tomography/computed tomography; CR, complete response; PR, partial response. ${ }^{a}$ Some patients also received radiation therapy after chemotherapy.

examination. However, given that Japanese people also have easy access to endoscopy through the screening program of their national health insurance system [15], the earlier age of diagnosis in Korea needs to be investigated further. Second, the predominance of female patients was noted in ours and the Japanese study unlike the European study [10,13]. This 
point should be clarified in future studies by comparing Western and Asian patients with duodenal-type FL. Third, the number of patients with abdominal pain was higher in the European study than in ours and the Japanese study, perhaps because most Asian cases were found as part of a regular health examination in the asymptomatic state. The histological grade and the distribution of stage were similar between the three studies, and most patients' risk on the IPI and FLIPI were scored as low (Table 2). When we analyzed the endoscopic findings of duodenal lesions, the main appearance was nodular or fine granular patterns consistent with previously observed findings [16].

The response to RT was excellent in the Japanese, European and our studies, and the response was maintained at the time of analysis. Thus, RT could be an effective initial treatment although there is no consensus on the best treatment option for duodenal-type FL. Considering that the diagnosis of lymphoma itself might give psychosocial stress to patients, RT could be the preferred option to a watch-andwait strategy. However, the RT dose was variable between the studies (Table 2). Since a randomized study comparing a standard dose (40-45 Gy in 20-23 fractions) with a low dose (24 Gy in 12 fractions) in indolent lymphomas showed no difference of response rate and survival [17], an RT dose of 24 Gy has been recommended for indolent lymphoma [18]. However, there is still insufficient data to confirm the efficacy of low-dose RT in duodenal-type FL. Our results with an RT dose of 24 Gy showed excellent outcomes comparable to that of Japanese and European studies using an RT dose of $30 \mathrm{~Gy}$ or greater $[10,13]$. Although the optimal target volume of RT is also uncertain, the International Lymphoma Radiation Oncology Group guideline recommends including the entire duodenum [18]. This is reasonable considering the very favorable clinical course of duodenal-type FL and increasing toxicity of a larger RT field. However, the need to extend the $\mathrm{RT}$ to the whole intestine still exists because duodenal-type FL often has multifocal lesions in the intestine [7].

Due to the nature of retrospective study, our study has a number of limitations. First, we did not perform a full evaluation for involvement of the small intestine. Thus, our staging work-up might have insufficiently reflected the exact amount of disease. However, considering the indolent nature of duodenal-type FL, it is not clear whether evaluation of the whole intestine would be beneficial for patients. Second, the median follow-up duration (20 months) was not sufficiently long to provide clear information about the occurrence of systemic relapse and transformation to large cell lymphoma. However, a previous study with a median follow-up of 77 months found no cases of systemic relapse or transformation among patients receiving RT [13]. Therefore, the risk of systemic progression or transformation might be minimal in patients with duodenal-type FL who were treated with RT. In addition, there is a lack of data about RT-induced longterm toxicities due to a short duration of follow-up.

In summary, duodenal-type FL had endoscopically unique findings and indolent clinical features. RT alone could effectively control duodenal lesion, although a further study with longer follow-up duration is warranted to confirm our findings.

\section{Electronic Supplementary Material}

Supplementary materials are available at Cancer Research and Treatment website (https://www.e-crt.org).

\section{Conflicts of Interest}

Conflict of interest relevant to this article was not reported.

\section{Acknowledgments}

This study was supported by a grant from the Basic Science Research Program through the National Research Foundation of Korea (NRF), which is funded by the Ministry of Education, Science, and Technology (NRF-2017R1A2B4005136).

\section{References}

1. Takata K, Okada H, Ohmiya N, Nakamura S, Kitadai Y, Tari A, et al. Primary gastrointestinal follicular lymphoma involving the duodenal second portion is a distinct entity: a multicenter, retrospective analysis in Japan. Cancer Sci. 2011;102: 1532-6.

2. Kim SJ, Choi CW, Mun YC, Oh SY, Kang HJ, Lee SI, et al. Multicenter retrospective analysis of 581 patients with primary intestinal non-hodgkin lymphoma from the Consortium for
Improving Survival of Lymphoma (CISL). BMC Cancer. 2011;11:321.

3. Swerdlow SH, Campo E, Pileri SA, Harris NL, Stein H, Siebert $\mathrm{R}$, et al. The 2016 revision of the World Health Organization classification of lymphoid neoplasms. Blood. 2016;127:237590.

4. Anderson JR, Armitage JO, Weisenburger DD. Epidemiology of the non-Hodgkin's lymphomas: distributions of the major 
subtypes differ by geographic locations. Non-Hodgkin's Lymphoma Classification Project. Ann Oncol. 1998;9:717-20.

5. Yamamoto S, Nakase H, Yamashita K, Matsuura M, Takada M, Kawanami C, et al. Gastrointestinal follicular lymphoma: review of the literature. J Gastroenterol. 2010;45:370-88.

6. Yoshino T, Miyake K, Ichimura K, Mannami T, Ohara N, Hamazaki S, et al. Increased incidence of follicular lymphoma in the duodenum. Am J Surg Pathol. 2000;24:688-93.

7. Takata K, Tanino M, Ennishi D, Tari A, Sato Y, Okada H, et al. Duodenal follicular lymphoma: comprehensive gene expression analysis with insights into pathogenesis. Cancer Sci. 2014;105:608-15.

8. Maeng CH, Ahn SW, Ryu SY, Han S, Ko YH, Ji JH, et al. Treatment outcomes and clinical relevance of the Follicular Lymphoma International Prognostic Index in Korean follicular lymphoma patients treated with chemotherapy. Korean J Intern Med. 2016;31:560-9.

9. Ahmed N, Owen TE, Rubinger M, Williams G, Nugent Z, Ahmed S, et al. Early stage W.H.O. grade I and II follicular lymphoma treated with radiation therapy alone. PLoS One. 2013;8:e65156.

10. Harada A, Oguchi M, Terui Y, Takeuchi K, Igarashi M, Kozuka T, et al. Radiation therapy for localized duodenal lowgrade follicular lymphoma. J Radiat Res. 2016;57:412-7.

11. Solal-Celigny P, Roy P, Colombat P, White J, Armitage JO, Arranz-Saez R, et al. Follicular lymphoma international prognostic index. Blood. 2004;104:1258-65.

12. Roulland S, Navarro JM, Grenot P, Milili M, Agopian J, Montpellier B, et al. Follicular lymphoma-like B cells in healthy individuals: a novel intermediate step in early lymphomagenesis. J Exp Med. 2006;203:2425-31.

13. Schmatz AI, Streubel B, Kretschmer-Chott E, Puspok A, Jager U, Mannhalter C, et al. Primary follicular lymphoma of the duodenum is a distinct mucosal/ submucosal variant of follicular lymphoma: a retrospective study of 63 cases. J Clin Oncol. 2011;29:1445-51.

14. Bende RJ, Smit LA, Bossenbroek JG, Aarts WM, Spaargaren M, de Leval L, et al. Primary follicular lymphoma of the small intestine: alpha4beta7 expression and immunoglobulin configuration suggest an origin from local antigen-experienced $B$ cells. Am J Pathol. 2003;162:105-13.

15. Leung WK, Wu MS, Kakugawa Y, Kim JJ, Yeoh KG, Goh KL, et al. Screening for gastric cancer in Asia: current evidence and practice. Lancet Oncol. 2008;9:279-87.

16. Iwamuro M, Kawai Y, Takata K, Kawano S, Yoshino T, Okada $\mathrm{H}$, et al. Primary intestinal follicular lymphoma: How to identify follicular lymphoma by routine endoscopy. World J Gastrointest Endosc. 2013;5:34-8.

17. Lowry L, Smith P, Qian W, Falk S, Benstead K, Illidge T, et al. Reduced dose radiotherapy for local control in non-Hodgkin lymphoma: a randomised phase III trial. Radiother Oncol. 2011;100:86-92.

18. Yahalom J, Illidge T, Specht L, Hoppe RT, Li YX, Tsang R, et al. Modern radiation therapy for extranodal lymphomas: field and dose guidelines from the International Lymphoma Radiation Oncology Group. Int J Radiat Oncol Biol Phys. 2015;92: 11-31. 